\title{
Pattern-avoiding Dyck paths $\rrbracket^{\dagger}$
}

\section{Antonio Bernini ${ }^{1} \quad$ Luca Ferrari $^{1} \quad$ Renzo Pinzani $^{1} \quad$ Julian West $^{2}$}

${ }^{1}$ University of Firenze, Dipartimento di Sistemi e Informatica, Italy

${ }^{2}$ Heilbronn Institute for Mathematical Research, University of Bristol, UK

\begin{abstract}
We introduce the notion of pattern in the context of lattice paths, and investigate it in the specific case of Dyck paths. Similarly to the case of permutations, the pattern-containment relation defines a poset structure on the set of all Dyck paths, which we call the Dyck pattern poset. Given a Dyck path $P$, we determine a formula for the number of Dyck paths covered by $P$, as well as for the number of Dyck paths covering $P$. We then address some typical pattern-avoidance issues, enumerating some classes of pattern-avoiding Dyck paths. Finally, we offer a conjecture concerning the asymptotic behavior of the sequence counting Dyck paths avoiding a generic pattern and we pose a series of open problems regarding the structure of the Dyck pattern poset.
\end{abstract}

Résumé. Nous proposons la notion d'un motif dans le contexte de chemins de treillis, et étudions le cas spécifique des chemins de Dyck. Comme dans le cas des permutations, on obtient une structure de poset sur l'ensemble de tous les chemins de Dyck, que nous appelons l'ensemble des chemins de Dyck partiellement ordonné selon le motif. Étant donné un chemin de Dyck $P$, nous déterminons une formule pour le nombre de chemins de Dyck couverts par $P$, ainsi que pour le nombre de chemins de Dyck couvrant $P$. Nous énumérons ensuite les chemins de Dyck évitant certaines catégories de motif. Enfin, nous proposons une conjecture asymptotique concernant le nombre de chemins de Dyck évitant un motif générique et nous posons quelques problèmes ouverts concernants la structure du poset etudié.

Keywords: Dyck path, pattern containment relation, enumeration

\section{Introduction}

One of the most investigated and fruitful notions in contemporary combinatorics is that of a pattern. Historically it was first considered for permutations [Kn], then analogous definitions were provided in the context of many other structures, such as set partitions [Go, K1, Sa], words [Bj, Bu] , and trees [DPTW, Gi, R]. Perhaps all of these examples have been motivated or informed by the more classical notion of graphs and subgraphs. Informally speaking, given a specific class of combinatorial objects, a pattern can be thought of as an occurrence of a small object inside a larger one; the word "inside" means that the pattern is suitably embedded into the larger object, depending on the specific combinatorial class of objects. The main aim of the present work is to introduce the notion of pattern in the context of lattice paths and to begin its systematic study in the special case of Dyck paths.

${ }^{\dagger}$ L. F. and R. P. partially supported by INdAM project: "Proprietà algebriche e combinatorie dei cammini discreti"; A. B. and L. F. partially supported by PRIN project: "Automi e Linguaggi Formali: Aspetti Matematici e Applicativi". 
For our purposes, a lattice path is a path in the discrete plane starting at the origin of a fixed Cartesian coordinate system, ending somewhere on the $x$-axis, never going below the $x$-axis and using only a prescribed set of steps $\Gamma$. We will refer to such paths as $\Gamma$-paths. This definition is extremely restrictive if compared to what is called a lattice path in the literature, but it will be enough for our purposes. Observe that a $\Gamma$-path can be alternatively described as a finite word on the alphabet $\Gamma$ obeying certain conditions. Using this language, we say that the length of a $\Gamma$-path is simply the length of the word which encodes such a path. Among the classical classes of lattice paths, the most common are those using only steps $U(p)=(1,1), D($ own $)=(1,-1)$ and $H$ (orizontal $)=(1,0)$; with these definitions, Dyck, Motzkin and Schröder paths correspond respectively to the set of steps $\{U, D\},\{U, H, D\}$ and $\left\{U, H^{2}, D\right\}$.

Consider the class $\mathcal{P}_{\Gamma}$ of all $\Gamma$-paths, for some choice of the set of steps $\Gamma$. Given $P, Q \in \mathcal{P}_{\Gamma}$ having length $k$ and $n$ respectively, we say that $Q$ contains (an occurrence of) the pattern $P$ whenever $P$ occurs as a subword of $Q$. So, for instance, in the class of Dyck paths, $U U D U D D U D U U D D$ contains the pattern $U U D D U D$, whereas in the class of Motzkin paths, $U U H D U U D H D D U D H U D$ contains the pattern $U H U D D H U D$. When $Q$ does not contain any occurrence of $P$ we will say that $Q$ avoids $P$. In the Dyck case, the previously considered path $U U D U D D U D U U D D$ avoids the pattern $U U U U D D D D$.

This notion of pattern gives rise to a partial order in a very natural way, by declaring $P \leq Q$ when $P$ occurs as a pattern in $Q$. In the case of Dyck paths, the resulting poset will be denoted by $\mathcal{D}$. It is immediate to notice that $\mathcal{D}$ has a minimum (the empty path), does not have a maximum, is locally finite and is ranked (the rank of a Dyck path is given by its semilength). As an example, we provide the Hasse diagram of an interval in the Dyck pattern poset:

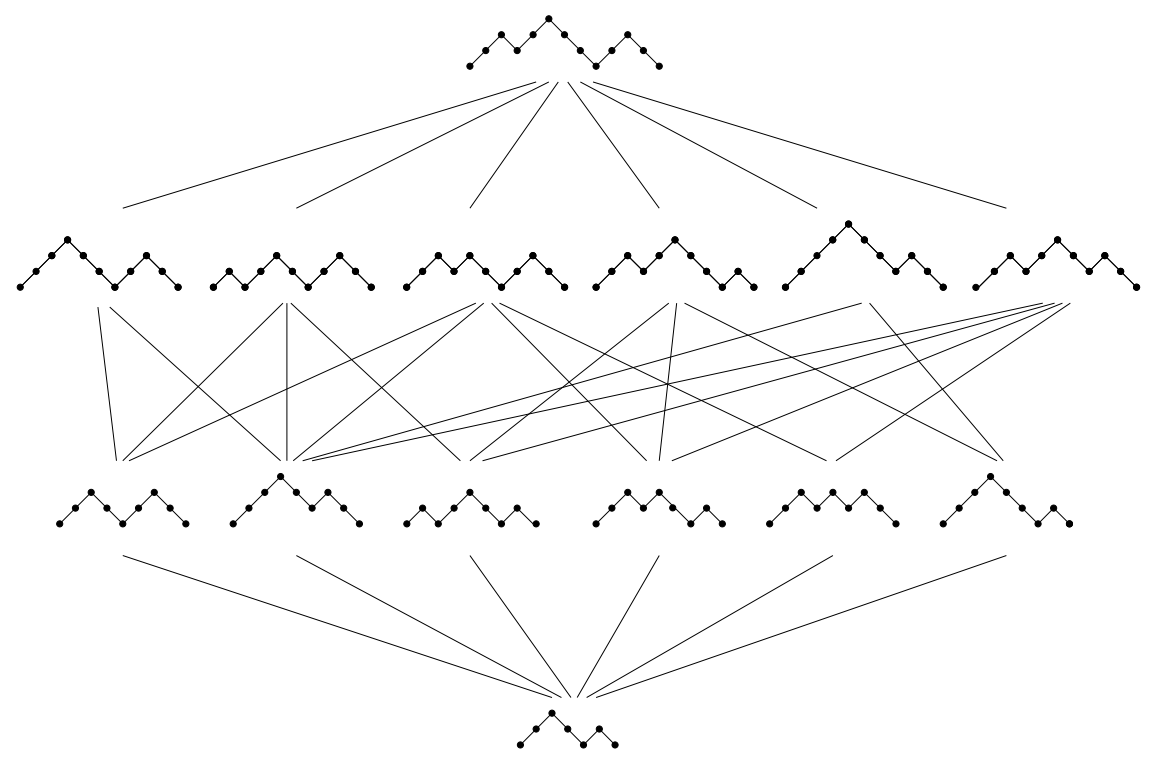

Observe that this notion of pattern for paths is very close to the analogous notion for words (considered, for instance, in [Bj], where the author determines the Möbius function of the associated pattern poset). Formally, instead of considering the set of all words of the alphabet $\{U, D\}$, we restrict ourselves to the 
set of Dyck words (so what we actually do is to consider a subposet of Björner's poset). However, the conditions a word has to obey in order to belong to this subposet (which translate into the fact of being a Dyck word) make this subposet highly nontrivial, and fully justify our approach, consisting of the study of its properties independently of its relationship with the full word pattern poset.

\section{The Dyck pattern poset}

In the Dyck pattern poset $\mathcal{D}$, following the usual notation for covering relation, we write $P \prec Q$ ( $Q$ covers $P$ ) to indicate that $P \leq Q$ and the rank of $P$ is one less than the rank of $Q$ (i.e., $\operatorname{rank}(P)=$ $\operatorname{rank}(Q)-1)$. Our first result concerns the enumeration of Dyck paths covered by a given Dyck path $Q$. We need some notation before stating it. Let $k+1$ be the number of points of $Q$ lying on the $x$-axis (call such points $p_{0}, p_{1}, \ldots, p_{k}$ ). Then $Q$ can be factorized into $k$ Dyck factors $F_{1}, \ldots, F_{k}$, each $F_{i}$ starting at $p_{i-1}$ and ending at $p_{i}$. Let $n_{i}$ be the number of ascents in $F_{i}$ (an ascent being a consecutive run of $U$ steps; $n_{i}$ also counts both the number of descents and the number of peaks in $F_{i}$ ). Moreover, we denote by $|U D U|$ and $|D U D|$ the number of occurrences in a Dyck path of a consecutive factor $U D U$ and $D U D$, respectively. In the path $Q$ of Figure 1 , we have $n_{1}=2, n_{2}=1, n_{3}=2,|U D U|=3$, and $|D U D|=2$.

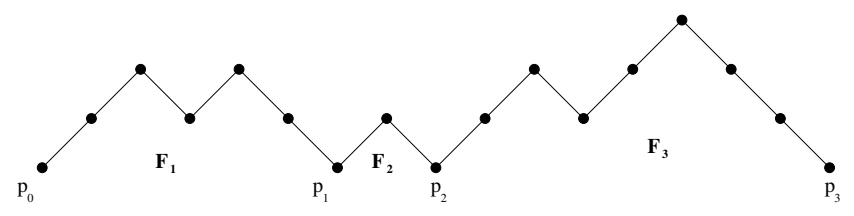

Fig. 1: A Dyck path having three factors.

Proposition 2.1 If $Q$ is a Dyck path with $k$ factors $F_{1}, \ldots F_{k}$, with $F_{i}$ having $n_{i}$ ascents, then the number of Dyck paths covered by $Q$ is given by

$$
\frac{\sum_{i=1}^{k} n_{i}^{2}+\left(\sum_{i=1}^{k} n_{i}\right)^{2}}{2}-|U D U|-|D U D|
$$

In a similar fashion, we are also able to find a formula for the number of all Dyck paths which cover a given path.

Proposition 2.2 If $Q$ is a Dyck path of semilength $n$ with $k$ factors $F_{1}, \ldots F_{k}$, with $F_{i}$ having semilength $f_{i}$, then the number of Dyck paths covering $Q$ is given by

$$
1+\sum_{i} f_{i}^{2}+\sum_{i<j} f_{i} f_{j}
$$

\section{Enumerative results on pattern-avoiding Dyck paths}

In the present section we will be concerned with the enumeration of some classes of pattern-avoiding Dyck paths. Similarly to what has been done for other combinatorial structures, we are going to consider 
classes of Dyck paths avoiding a single pattern, and we will examine the cases of short patterns. Specifically, we will count Dyck paths avoiding any single path of length $\leq 3$; each case will arise as a special case of a more general result concerning a certain class of patterns.

Given a pattern $P$, we denote by $D_{n}(P)$ the set of all Dyck paths of semilength $n$ avoiding the pattern $P$, and by $d_{n}(P)$ the cardinality of $D_{n}(P)$.

\subsection{The pattern $(U D)^{k}$}

This is one of the easiest cases.

Proposition 3.1 For any $k \in \mathbf{N}, Q \in D_{n}\left((U D)^{k}\right)$ if and only if $Q$ has at most $k-1$ peaks.

Since it is well known that the number of Dyck paths of semilength $n$ and having $k$ peaks is given by the Narayana number $N_{n, k}$ (sequence A001263 in [S1]), we have that $d_{n}\left((U D)^{k}\right)=\sum_{i=0}^{k-1} N_{n, i}$ (partial sums of Narayana numbers). Thus, in particular:

$$
\begin{aligned}
& \text { - } d_{n}(U D)=0 \\
& \text { - } d_{n}(U D U D)=1 \\
& \text { - } d_{n}(U D U D U D)=1+\left(\begin{array}{l}
n \\
2
\end{array}\right) .
\end{aligned}
$$

\subsection{The pattern $U^{k-1} D U D^{k-1}$}

Let $Q$ be a Dyck path of length $2 n$ and $P=U^{k-1} D U D^{k-1}$. Clearly if $n<k$, then $Q$ avoids $P$, and if $n=k$, then all Dyck paths of length $2 n$ except one ( $Q$ itself) avoid $Q$. Therefore:

- $d_{n}(P)=C_{n}$ if $n<k$, and

- $d_{n}(P)=C_{n}-1$ if $n=k$,

where $C_{n}$ is the $n$-th Catalan number.

Now suppose $n>k$. Denote by $A$ the end point of the $(k-1)$-th $U$ step of $Q$. It is easy to verify that $A$ belongs to the line $r$ having equation $y=-x+2 k-2$. Denote with $B$ the starting point of the $(k-1)$-th-to-last $D$ step of $Q$. An analogous computation shows that $B$ belongs to the line $s$ having equation $y=x-(2 n-2 k+2)$.

Depending on how the two lines $r$ and $s$ intersect, it is convenient to distinguish two cases.

1. If $2 n-2 k+2 \geq 2 k-4$ (i.e. $n \geq 2 k-3$ ), then $r$ and $s$ intersect at height $\leq 1$, whence $x_{A} \leq x_{B}$ (where $x_{A}$ and $x_{B}$ denote the abscissas of $A$ and $B$, respectively). The path $Q$ can be split into three parts (see Figure 2): a prefix $Q_{A}$ from the origin $(0,0)$ to $A$, a path $X$ from $A$ to $B$, and a suffix $Q_{B}$ from $B$ to the last point $(2 n, 0)$.

We point out that $Q_{A}$ has exactly $k-1 U$ steps and its last step is a $U$ step. Analogously, $Q_{B}$ has exactly $k-1 D$ steps and its first step is a $D$ step. Notice that there is a clear bijection between the set $\mathcal{A}$ of Dyck prefixes having $k-1 U$ steps and ending with a $U$ and the set $\mathcal{B}$ of Dyck suffixes having $k-1 D$ steps and starting with a $D$, since each element of $\mathcal{B}$ can be read from right to left thus obtaining an element of $\mathcal{A}$. Moreover, $\mathcal{A}$ is in bijection with the set of Dyck paths of semilength $k-1$ (just complete each element of $\mathcal{A}$ with the correct sequence of $D$ steps), hence $|\mathcal{A}|=C_{k-1}$. 


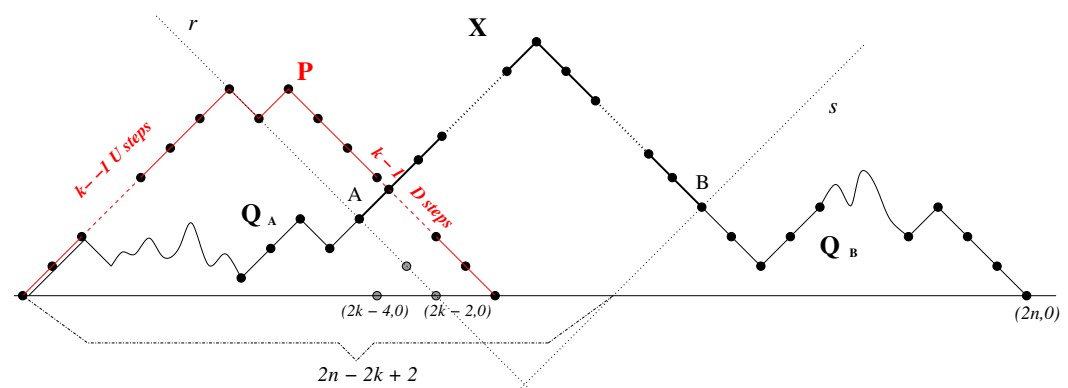

Fig. 2: Avoiding $U^{k-1} D U D^{k-1}$, with $n \geq 2 k-3$

If we require $Q$ to avoid $P$, then necessarily $X=U^{i} D^{j}$, for suitable $i, j$ (for, if a valley $D U$ occurred in $X$, then $Q$ would contain $P$ since $U^{k-1}$ and $D^{k-1}$ already occur in $Q_{A}$ and $Q_{B}$, respectively). In other words, $A$ and $B$ can be connected only in one way, using a certain number (possibly zero) of $U$ steps followed by a certain number (possibly zero) of $D$ steps. Therefore, a path $Q$ avoiding $P$ is essentially constructed by choosing a prefix $Q_{A}$ from $\mathcal{A}$ and a suffix $Q_{B}$ from $\mathcal{B}$, whence:

$$
d_{n}(P)=C_{k-1}^{2}, \quad(\text { if } \quad n \geq 2 k-3) .
$$

2. Suppose now $k+1 \leq n<2 k-3$ (which means that $r$ and $s$ intersect at height $>1$ ). Then it can be either $x_{A} \leq x_{B}$ or $x_{A}>x_{B}$.

a) If $x_{A} \leq x_{B}$, then we can count all Dyck paths $Q$ avoiding $P$ using an argument analogous to the previous one. However, in this case the set of allowable prefixes of each such $Q$ is a proper subset of $\mathcal{A}$. More specifically, we have to consider only those for which $x_{A}=$ $k-1, k, k+1, \ldots, n$ (see Figure 3). In other words, an allowable prefix has $k-1 U$ steps

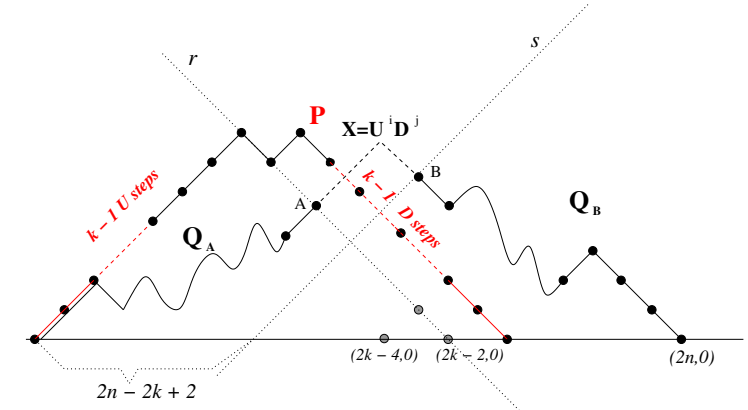

Fig. 3: Avoiding $U^{k-1} D U D^{k-1}$, with $x_{A} \leq x_{B}$

and $0,1,2, \ldots$ or $n-k+1 D$ steps. If $b_{i, j}$ denotes the numbers of Dyck prefixes with $i U$ 
steps and $j D$ steps $\left(i \geq j\right.$ ), then the contribution to $d_{n}(P)$ in this case is

$$
d_{n}^{(1)}(P)=\left(\sum_{j=0}^{n-k+1} b_{k-2, j}\right)^{2}
$$

The coefficients $b_{i, j}$ are the well-known ballot numbers (sequence A009766 in [S1]), whose first values are reported in Table 1 .

b) If $x_{A}>x_{B}$, then it is easy to see that $Q$ necessarily avoids $P$, since $A$ clearly occurs after $B$, and so there are strictly less than $k-1 D$ steps from $A$ to $(2 n, 0)$. Observe that, in this case, the path $Q$ lies below the profile drawn by the four lines $y=x, r, s$ and $y=-x+2 n$. In order to count these paths, referring to Figure 4, just split each of them into a prefix and a suffix of equal length $n$ and call $C$ the point having abscissa $n$.

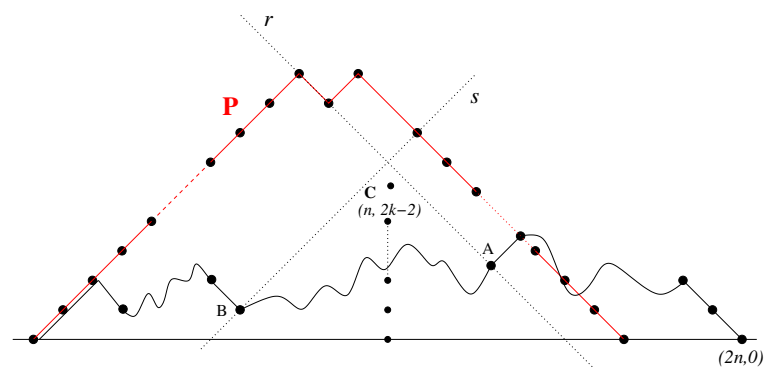

Fig. 4: Avoiding $U^{k-1} D U D^{k-1}$, with $x_{A}>x_{B}$

Since $C$ must lie under the point where $r$ and $s$ intersect, then its ordinate $y_{C}$ equals $-n+$ $2 k-2-2 t$ with $t \geq 1$ (and also recalling that $y_{C}=-n+2 k-2-2 t \geq 0$ ). A prefix whose final point is $C$ has $k-j U$ steps and $n-k+j D$ steps, with $j \geq 2$. Since, in this case, a path $Q$ avoiding $P$ is constructed by gluing a prefix and a suffix chosen among $b_{k-j, n-k+j}$ possibilities $(j \geq 2)$, we deduce that the contribution to $d_{n}(P)$ in this case is:

$$
d_{n}^{(2)}(P)=\sum_{j \geq 2} b_{k-j, n-k+j}^{2}
$$

Summing up the two contributions we have obtained in a) and b), we get:

$$
\begin{aligned}
d_{n}(P) & =d_{n}^{(1)}(P)+d_{n}^{(2)}(P) \\
& =\left(\sum_{j=0}^{n-k+1} b_{k-2, j}\right)^{2}+\sum_{j \geq 2} b_{k-j, n-k+j}^{2}, \quad \text { if } \quad k+1 \leq n<2 k-3 .
\end{aligned}
$$




\begin{tabular}{|c|c|c|c|c|c|c|c|c|c|c|}
\hline 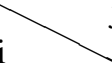 & 0 & 1 & 2 & 3 & 4 & 5 & 6 & 7 & 8 & 9 \\
\hline 0 & 1 & & & & & & & & & \\
\hline 1 & 1 & 1 & & & & & & & & \\
\hline 2 & 1 & 2 & 2 & & & & & & & \\
\hline 3 & 1 & 3 & 5 & 5 & & & & & & \\
\hline 4 & 1 & 4 & 9 & 14 & 14 & & & & & \\
\hline 5 & 1 & 5 & 14 & 28 & 42 & 42 & & & & \\
\hline 6 & 1 & 6 & 20 & 48 & 90 & 132 & 132 & & & \\
\hline 7 & 1 & 7 & 27 & 75 & 165 & 297 & 429 & 429 & & \\
\hline 8 & 1 & 8 & 35 & 110 & 275 & 572 & 1001 & 1430 & 1430 & \\
\hline 9 & 1 & 9 & 44 & 154 & 429 & 1001 & 2002 & 3432 & 4862 & 4862 \\
\hline
\end{tabular}

Tab. 1: The sum of the gray entries gives the bold entry in the line below. The sum of the squares of the bold entries gives an appropriate element of Table 2

Notice that formula (4) reduces to the first sum if $n \geq 2 k-3$, since in that case $n-k+j>k-j$, for $j \geq 2$. We then have a single formula including both cases 1 . and 2. :

$$
d_{n}(P)=\left(\sum_{j=0}^{n-k+1} b_{k-2, j}\right)^{2}+\sum_{j \geq 2} b_{k-j, n-k+j}^{2}, \quad \text { if } \quad n \geq k+1
$$

Formula $(5)$ can be further simplified by recalling a well known recurrence for ballot numbers, namely that, when $j \leq i+1$,

$$
b_{i+1, j}=\sum_{s=0}^{j} b_{i, s} .
$$

Therefore, we get the following interesting expression for $d_{n}(P)$ (when $n \geq k+1$ ) in terms of sums of squares of ballot numbers along a skew diagonal (see also Tables 1]and 2):

$$
d_{n}(P)=\left\{\begin{array}{cc}
C_{k-1}^{2} & \text { if } n \geq 2 k-3 \\
\sum_{j \geq 1} b_{k-j, n-k+j}^{2} & \text { otherwise. }
\end{array}\right.
$$

Therefore we obtain in particular:

$$
d_{n}(U U D U D D)=4 \text {, when } n \geq 3 .
$$

\subsection{The pattern $U^{k} D^{k}$}

The case $P=U^{k} D^{k}$ is very similar to the previous one. We just observe that, when $x_{A} \leq x_{B}$, the two points $A$ and $B$ can be connected only using a sequence of $D$ steps followed by a sequence of $U$ steps. This is possible only if $n \leq 2 k-2$, which means that $r$ and $s$ do not intersect below the $x$-axis. Instead, if $n \geq 2 k-1, Q$ cannot avoid $P$. Therefore we get (see also Table 3 ):

$$
d_{n}(P)=\left\{\begin{array}{cc}
0 & \text { if } n \geq 2 k-1 \\
\sum_{j \geq 1} b_{k-j, n-k+j}^{2} & \text { otherwise }
\end{array}\right.
$$




\begin{tabular}{cc|cccccccccccccccccc}
\hline$k$ & $n$ & 0 & 1 & 2 & 3 & 4 & 5 & 6 & 7 & 8 & 9 & 10 & 11 & 12 & 13 \\
\hline 1 & 0 & 0 & 0 & 0 & 0 & 0 & 0 & 0 & 0 & 0 & 0 & 0 & 0 & $\ldots$ \\
2 & 1 & 1 & 1 & 1 & 1 & 1 & 1 & 1 & 1 & 1 & 1 & 1 & 1 & 1 \\
3 & 1 & 1 & 2 & 4 & 4 & 4 & 4 & 4 & 4 & 4 & 4 & 4 & 4 & 4 & $\ldots$ \\
4 & 1 & 1 & 2 & 5 & 13 & 25 & 25 & 25 & 25 & 25 & 25 & 25 & 25 & 25 & $\ldots$ & 196 & $\ldots$ & 196 \\
5 & 1 & 1 & 2 & 5 & 14 & 41 & $\mathbf{1 0 6}$ & 196 & 196 & 196 & 196 & 196 & 196 & 1764 & $\ldots$ \\
6 & 1 & 1 & 2 & 5 & 14 & 42 & 131 & $\mathbf{3 9 2}$ & $\mathbf{9 8 0}$ & 1764 & 1764 & 1764 & 1764 & 1764 \\
7 & 1 & 1 & 2 & 5 & 14 & 42 & 132 & 428 & $\mathbf{1 3 8 0}$ & $\mathbf{4 0 6 8}$ & $\mathbf{9 8 6 4}$ & 17424 & 17424 & 17424 & $\ldots$ \\
8 & 1 & 1 & 2 & 5 & 14 & 42 & 132 & 429 & 1429 & $\mathbf{4 7 9 7}$ & $\mathbf{1 5 4 8 9}$ & $\mathbf{4 4 6 4 9}$ & $\mathbf{1 0 5 6 3 3}$ & 184041 & $\ldots$ \\
9 & 1 & 1 & 2 & 5 & 14 & 42 & 132 & 429 & 1430 & 4861 & $\mathbf{1 6 7 1 4}$ & $\mathbf{5 6 7 4 9}$ & $\mathbf{1 8 1 2 5 8}$ & $\mathbf{5 1 1 2 2 5}$ & $\ldots$
\end{tabular}

Tab. 2: Number of Dyck paths of semilength $n$ avoiding $U^{k-1} D U D^{k-1}$. Entries in boldface are the nontrivial ones $(k+1 \leq n<2 k-3)$.

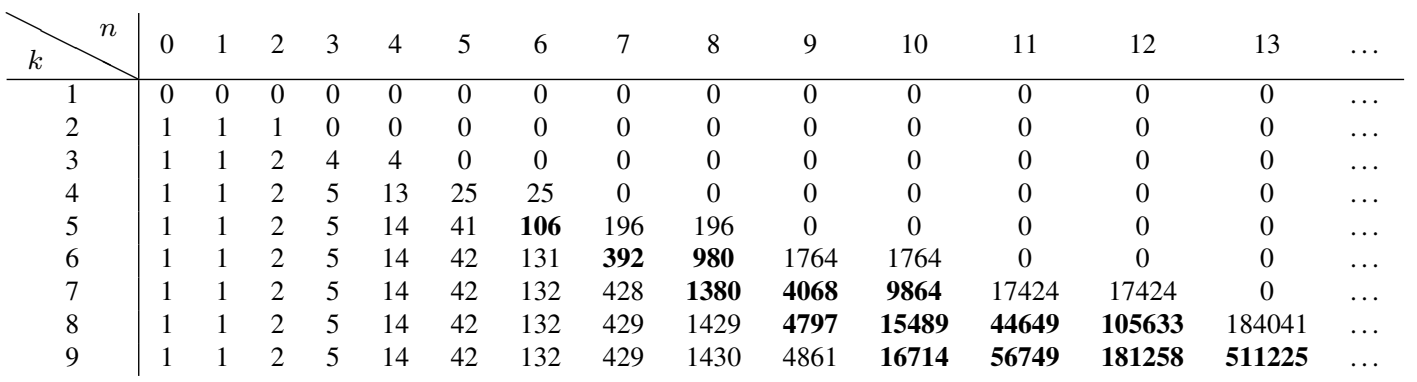

Tab. 3: Number of Dyck paths of semilength $n$ avoiding $U^{k} D^{k}$. Entries in boldface are the nontrivial ones $(k+1 \leq$ $n<2 k-3)$.

In particular, we then find:

- $d_{n}(U U D D)=0$, when $n \geq 3$;

- $d_{n}(U U U D D D)=0$, when $n \geq 5$.

\subsection{The pattern $U^{k-1} D^{k-1} U D$}

This is by far the most challenging case.

Let $Q$ be a Dyck path of length $2 n$ and $P=U^{k-1} D^{k-1} U D$. If $Q$ avoids $P$, then there are two distinct options: either $Q$ avoids $U^{k-1} D^{k-1}$ or $Q$ contains such a pattern. In the first case, we already know that $d_{n}\left(U^{k-1} D^{k-1}\right)$ is eventually equal to zero. So, for the sake of simplicity, we will just find a formula for $d_{n}(P)$ when $n$ is sufficiently large, i.e. $n \geq 2 k-3$. Therefore, for the rest of this section, we will suppose that $Q$ contains $U^{k-1} D^{k-1}$.

The $(k-1)$-th $D$ step of the first occurrence of $U^{k-1} D^{k-1}$ in $Q$ lies on the line having equation $y=-x+2 n$. This is due to the fact that $Q$ has length $2 n$ and there cannot be any occurrence of $U D$ after the first occurrence of $U^{k-1} D^{k-1}$. The path $Q$ touches the line of equation $y=-x+2 k-2$ for the first time with the end point $A$ of its $(k-1)$-th $U$ step. After that, the path $Q$ must reach the starting point $B$ of the $(k-1)$-th $D$ step occurring after $A$. Finally, a sequence of consecutive $D$ steps terminates $Q$ (see Figure 5). 
Therefore, $Q$ can be split into three parts: the first part, from the beginning to $A$, is a Dyck prefix having $k-1 U$ steps and ending with a $U$ step; the second part, from $A$ to $B$, is a path using $n-k+1 U$ steps and $k-2 D$ steps; and the third part, from $B$ to the end, is a sequence of $D$ steps (whose length depends on the coordinates of $A$ ). However, both the first and the second part of $Q$ have to obey some additional constraints.

The height of the point $A$ (where the first part of $Q$ ends) must allow $Q$ to have at least $k-1 D$ steps after $A$. Thus, the height of $A$ plus the number of $U$ steps from $A$ to $B$ minus the number of $D$ steps from $A$ to $B$ must be greater than or equal to 1 (to ensure that the pattern $U^{k-1} D^{k-1}$ occurs in $Q$ ). Hence, denoting with $x$ the maximum number of $D$ steps which can occur before $A$, either $x=k-2$ or the following equality must be satisfied:

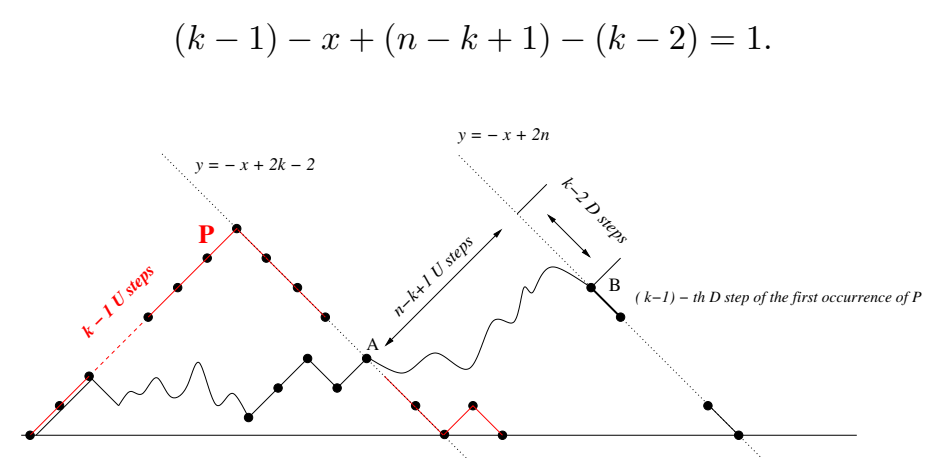

Fig. 5: A path $Q$ avoiding $P=U^{k-1} D^{k-1} U D$

Therefore, $x=\min \{n-k+1, k-2\}$. Observe however that, since we are supposing that $n \geq 2 k-3$, we always have $x=k-2$.

Concerning the part of $Q$ between $A$ and $B$, since we have to use $n-k+1 U$ steps and $k-2 D$ steps, there are $\left(\begin{array}{l}n-1 \\ k-2\end{array}\right)$ distinct paths connecting $A$ and $B$. However, some of them must be discarded, since they fall below the $x$-axis. In order to count these "bad" paths, we split each of them into two parts. Namely, if $A^{\prime}$ and $B^{\prime}$ are the starting and ending points of the first (necessarily $D$ ) step below the $x$-axis, the part going from $A$ to $A^{\prime}$, and the remaining part (see Fig. 6).

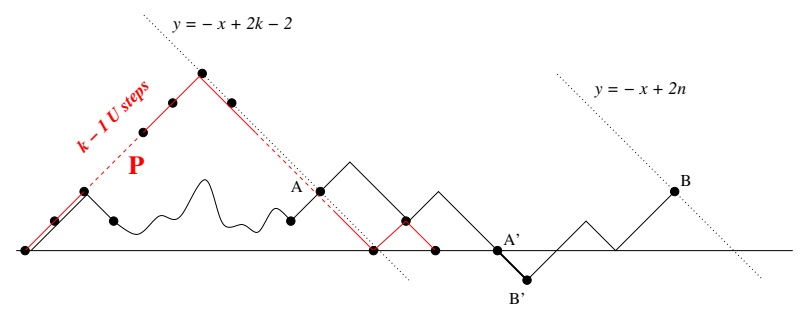

Fig. 6: A forbidden subpath from $A$ to $B$.

It is not too hard to realize that the number of possibilities we have to choose the first part is given 


\begin{tabular}{cc|cccccccccc}
\hline$k$ & $n$ & 0 & 1 & 2 & 3 & 4 & 5 & 6 & 7 & 8 & 9 \\
\hline 1 & & 1 & 1 & 0 & 0 & 0 & 0 & 0 & 0 & 0 & 0 \\
2 & 1 & 1 & 1 & 1 & 1 & 1 & 1 & 1 & 1 & 1 \\
3 & 1 & 1 & 2 & 4 & 6 & 8 & 10 & 12 & 14 & 16 \\
4 & 1 & 1 & 2 & 5 & 13 & 28 & 48 & 73 & 103 & 138 \\
5 & 1 & 1 & 2 & 5 & 14 & 41 & 110 & 245 & 450 & 739 \\
6 & 1 & 1 & 2 & 5 & 14 & 42 & 131 & 397 & 1069 & 2427
\end{tabular}

Tab. 4: Avoiding $U^{k-1} D^{k-1} U D$

by a ballot number (essentially because, reading the path from right to left, we have to choose a Dyck prefix from $A^{\prime}$ to $A$ ), whereas the number of possibilities we have to choose the second part is given by a binomial coefficient (essentially because, after having discarded the step starting at $A^{\prime}$, we have to choose an unrestricted path from $B^{\prime}$ to $B$ ). After a careful inspection, we thus get to the following expression for the total number $d_{n}(P)$ of Dyck paths of semilength $n \geq 2 k-3$ avoiding $P$ :

$$
\begin{aligned}
d_{n}(P)= & \left(\begin{array}{l}
n-1 \\
k-2
\end{array}\right) C_{k-1} \\
& -\sum_{s=2}^{k-2} b_{k-2, s} \cdot\left(\sum_{i=0}^{s-2} b_{k-3-i, s-2-i}\left(\begin{array}{c}
n-k-s+3+2 i \\
i
\end{array}\right)\right) .
\end{aligned}
$$

Formula (7) specializes to the following expressions for low values of $k$ (see also Table 4 ):

- when $k=3, d_{n}(P)=2 n-2$ for $n \geq 3$;

- when $k=4, d_{n}(P)=\frac{5 n^{2}-15 n+6}{2}$ for $n \geq 5$;

- when $k=5, d_{n}(P)=\frac{14 n^{3}-84 n^{2}+124 n-84}{6}$ for $n \geq 7$.

\section{Some remarks on the asymptotics of pattern-avoiding Dyck paths}

In this final section we collect some thoughts concerning the asymptotic behavior of integer sequences counting pattern-avoiding Dyck paths. Unlike the case of permutations, for Dyck paths it seems plausible that a sort of "master theorem" exists, at least in the case of single avoidance. This means that all the sequences which count Dyck paths avoiding a single pattern $P$ have the same asymptotic behavior (with some parameters, such as the leading coefficient, depending on the specific path $P$ ). We have some computational evidence which leads us to formulate a conjecture, whose proof we have not been able to complete, and so we leave it as an open problem.

Let $P$ denote a fixed Dyck path of semilength $x$. We are interested in the behavior of $d_{n}(P)$ when $n \rightarrow \infty$. Our conjecture is the following: 
Conjecture. Suppose that $P$ starts with a $U$ steps and ends with $b D$ steps. Then, setting $k=2 x-$ $2-a-b$, we have that $d_{n}(P)$ is asymptotic to

$$
\frac{\alpha_{P} \cdot C_{a} \cdot C_{b}}{k !} n^{k},
$$

where $C_{m}$ denotes the $m$-th Catalan numbers and $\alpha_{P}$ is the number of saturated chains in the Dyck lattice of order $x$ (see $[[F P])$ from $P$ to the maximum $U^{x} D^{x}$.

Equivalently, $\alpha_{P}$ is the number of standard Young tableaux whose Ferrers shape is determined by the region delimited by the path $P$ and the path $U^{x} D^{x}$, as shown in Figure 7

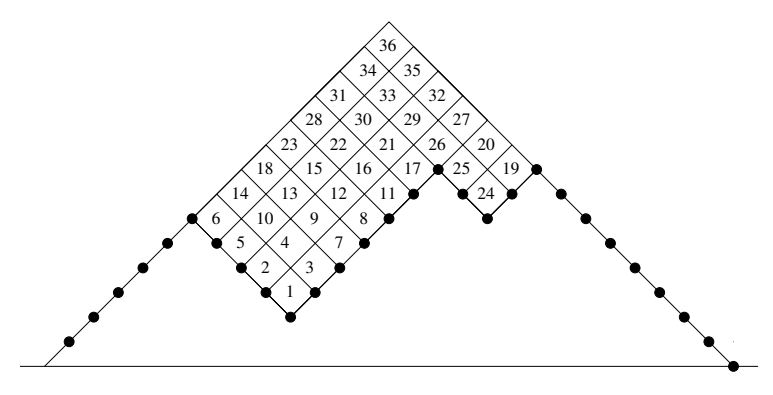

Fig. 7: The standard Young tableau determined by a Dyck path.

We close our paper with some further conjectures concerning the order structure of the Dyck pattern poset.

- What is the Möbius function of the Dyck pattern poset (from the bottom element to a given path? Of a generic interval?)?

- How many (saturated) chains are there up to a given path? Or in a general interval?

- Does there exist an infinite antichain in the Dyck pattern poset?

The last conjecture has been suggested by an analogous one for the permutation pattern poset which has been solved in the affirmative (see $[\overline{\mathrm{SB}}]$ and the accompanying comment). In the present context we have no intuition on what could be the answer, though we are a little bit less optimistic than in the permutation case.

\section{References}

[Bj] A. Björner, The Möbius function of subword order, Invariant theory and tableaux (Minneapolis, MN, 1988), 118-124, IMA Vol. Math. Appl., 19, Springer, New York, 1990.

[Bu] A. Burstein, Enumeration of words with forbidden patterns, $\mathrm{PhD}$ thesis, University of Pennsylvania, 1998. 
[DPTW] M. Dairyko, L. Pudwell, S. Tyner, C. Wynn, Non-contiguous pattern avoidance in binary trees, Electron. J. Combin., 19(3) (2012) \#P22.

[FP] L. Ferrari, R. Pinzani, Lattices of lattice paths, J. Statist. Plann. Inference, 135 (2005) 77-92.

[Gi] Samuele Giraudo, Balanced binary trees in the Tamari lattice, Discrete Math. Theoret. Comput. Sci. Proc., AN (2010) 593-604.

[Go] A. Goyt, Avoidance of partitions of a three element set, Adv. Appl. Math., 41 (2008) 95-114.

[K1] M. Klazar, On abab-free and abba-free sets partitions, European J. Combin., 17 (1996) 53-68.

[Kn] D. Knuth, The art of computer programming, Vol. 1, Addison Wesley, Boston, 1968.

[R] E. Rowland, Pattern avoidance in binary trees, J. Combin. Theory Ser. A, 117 (2010) 741-758.

[Sa] B. E. Sagan, Pattern avoidance in set partitions, Ars Combin., 94 (2010) 79-96.

[SI] N. J. A. Sloane, The On-Line Encyclopedia of Integer Sequences, electronically available at oeis.org.

[SB] D. A. Spielman, M. Bóna, An infinite antichain of permutations, Electron. J. Combin., 7 (2000) \#N2 (4 pp.). 